\title{
Electroluminescence from a diamond device with ion-beam-micromachined buried graphitic electrodes
}

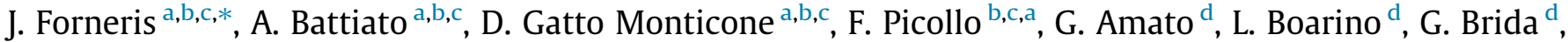 \\ I. P. Degiovanni ${ }^{d}$, E. Enrico ${ }^{d}$, M. Genovese ${ }^{d}$, E. Moreva $^{d}$, P. Traina ${ }^{d}$, C. Verona $^{\mathrm{e}}$, G. Verona Rinati $^{\mathrm{e}}$, \\ P. Olivero ${ }^{\mathrm{a}, \mathrm{b}, \mathrm{c}}$
}

\footnotetext{
${ }^{a}$ Physics Department and NIS Interdepartmental Centre, University of Torino, Torino, Italy

${ }^{\mathrm{b}}$ Istituto Nazionale di Fisica Nucleare (INFN), Sez. Torino, Torino, Italy

${ }^{c}$ Consorzio Nazionale Interuniversitario per le Scienze Fisiche della Materia (CNISM), Sez. Torino, Torino, Italy

${ }^{\mathrm{d}}$ Istituto Nazionale di Ricerca Metrologica (INRiM), Torino, Italy

e Department of Industrial Engineering, University of Roma "Tor Vergata", Roma, Italy
}

\section{A R T I C L E I N F O}

\section{Article history:}

Received 10 July 2014

Received in revised form 12 December 2014

Accepted 15 December 2014

Available online 6 January 2015

\section{Keywords:}

Diamond

Electroluminescence

Graphite

Ion beam micro-machining

\begin{abstract}
A B S T R A C T
Focused $\mathrm{MeV}$ ion microbeams are suitable tools for the direct writing of conductive graphitic channels buried in an insulating diamond bulk, as demonstrated in previous works with the fabrication of multi-electrode ionizing radiation detectors and cellular biosensors. In this work we investigate the suitability of the fabrication method for the electrical excitation of color centers in diamond. Differently from photoluminescence, electroluminescence requires an electrical current flowing through the diamond sub-gap states for the excitation of the color centers. With this purpose, buried graphitic electrodes with a spacing of $10 \mu \mathrm{m}$ were fabricated in the bulk of a detector-grade CVD single-crystal diamond sample using a scanning $1.8 \mathrm{MeV} \mathrm{He}^{+}$micro-beam. The current flowing in the gap region between the electrodes upon the application of a $450 \mathrm{~V}$ bias voltage was exploited as the excitation pump for the electroluminescence of different types of color centers localized in the above-mentioned gap. The bright light emission was spatially mapped using a confocal optical microscopy setup. The spectral analysis of electroluminescence revealed the emission from neutrally-charged nitrogen-vacancy centers $\left(\mathrm{NV}^{0}, \lambda_{\mathrm{ZPL}}=575 \mathrm{~nm}\right)$, as well as from cluster crystal dislocations (A-band, $\lambda=400-500 \mathrm{~nm}$ ). Moreover, an electroluminescence signal with appealing spectral features (sharp emission at room temperature, low phonon sidebands) from He-related defects was detected $\left(\lambda_{\mathrm{ZPL}}=536.3 \mathrm{~nm}, \lambda_{\mathrm{ZPL}}=560.5 \mathrm{~nm}\right)$; a low and broad peak around $\lambda=740 \mathrm{~nm}$ was also observed and tentatively ascribed to Si-V or GR1 centers. These results pose interesting future perspectives for the fabrication of electrically-stimulated single-photon emitters in diamond for applications in quantum optics and quantum cryptography.
\end{abstract}

(c) 2014 Elsevier B.V. All rights reserved.

\section{Introduction}

Color centers in diamond are attracting an ever-growing interest due to their appealing photon-emission properties (high stability and quantum efficiency at room temperature) for applications in quantum optics and photonics [1].

The development of diamond devices based on the functionalities of sub-superficial graphitic micro-electrodes has been investigated in several recent works [2,3]. The fabrication method relies on the direct writing in the single-crystal diamond bulk of

* Corresponding author at: Physics Department, University of Torino, via P. Giuria, 1-10126 Torino, Italy.

E-mail address: jacopo.forneris@unito.it (J. Forneris). amorphized channels by the selective damage induction associated with the Bragg's peak of $\mathrm{MeV}$ ions. The crystal volume in which the radiation-induced vacancy density exceeds a threshold value converts to nano-crystalline graphite upon thermal treatment at temperatures above $900{ }^{\circ} \mathrm{C}$, while the diamond lattice is partially recovered where the radiation damage density is lower $[4,5]$.

Since the radiation damage from $\mathrm{MeV}$ ions is prominently induced at the ion end of range, the fabrication method has been proved to be effective for the fabrication of conductive graphitic electrodes located several micrometers below the surface of the electrically insulating and optically transparent diamond dielectric. The fabrication process is characterized by a spatial resolution which is limited only by the ion beam spot size and by the ion lateral straggling in the material. The technique has been effectively 
exploited to fabricate and characterize diamond-based devices such as ionizing radiation detectors [3,6] cellular bio-sensors [7] and IR emitters [8].

In this work, we investigate the potential exploitation of the fabrication technique for the electrical excitation of color centers in diamond, aiming at the development of practical single-photon-emitting devices based on the efficiency and stability of the above-mentioned centers, for applications in quantum optics $[9,10]$.

With this purpose, a diamond device with four independent buried graphitic electrodes was fabricated by means of $\mathrm{MeV}$ ion beam lithography. Differently from previous works, in which the electrical excitation of color centers is achieved by means of diodes and p-i-n structures [9-11], the device proposed in this work exploits the current flowing between buried graphitic electrodes, in order to obtain electroluminescence (EL) from the diamond region located in the inter-electrode gap. The light emission properties of the fabricated structure were investigated by means of luminescence mapping and the relevant spectra were analyzed to attribute the active defects in the device under investigation.

\section{Experimental}

\subsection{Sample preparation}

The device under test, whose IBIC characterization was previously reported in [6], is based on a $\sim 40 \mu \mathrm{m}$ thick intrinsic diamond film homoepitaxially grown on a commercially available $4 \times 4 \times 0.4 \mathrm{~mm}^{3}$ type-Ib single-crystal high-pressure high-temperature (HPHT) substrate, using a microwave plasma enhanced chemical vapor deposition process at the laboratories of Rome "Tor Vergata" University. A scanning $1.8 \mathrm{MeV} \mathrm{He}^{+}$ion microbeam ( $\sim 10 \mu \mathrm{m}$ spot size) was used to perform a deep ion beam lithography (DIBL) process $[2,6]$ at the AN2000 micro-beam line of the INFN National Laboratories of Legnaro (I). Five graphitic channels were written at a depth of $\sim 3 \mu \mathrm{m}$ below the diamond surface using a ion fluence of $\sim 1.5 \times 10^{17} \mathrm{~cm}^{-2}$, which, according to SRIM2011 simulations [12], is sufficient to achieve a vacancy density above the graphitization threshold at the end of range (Fig. 1a) [6].

Before the DIBL process, a slowly thinning copper mask was deposited on the diamond surface in order to control the depth of the $1.8 \mathrm{MeV} \mathrm{He}^{+}$Bragg's peak in diamond, ensuring the emersion of the buried graphitic channels at their endpoints, as described in previous works $[2,3,6]$.
The implanted sample underwent a thermal annealing process for $2 \mathrm{~h}$ in vacuum, in order to promote the conversion of the amorphized regions at the end of the Bragg's peak to a graphitic phase and to concurrently recover the residual structural damage in the region comprised between the buried channels and the diamond surface. The resulting micro-fabricated structure (Fig. 1b) consisted of four parallel, $\sim 10 \mu \mathrm{m}$ wide independent graphitic electrodes, spaced by $\sim 12 \mu \mathrm{m}$, plus an additional horizontal electrode, which was not employed in the present work. Each graphitic channel was connected to the external circuitry by the deposition of $80 \mathrm{~nm}$ thick $\mathrm{Cr} / \mathrm{Al}$ circular contacts $(150 \mu$ m diameter $)$, which were exploited as pads for the electrodes wire-bonding. As schematically represented in Fig. 1b, the graphitic channels were shorted in pairs, defining a two-electrode inter-digitated geometry. Due to the ion micro-beam spot size and to the absence of an implantation mask, stray ions were implanted in the inter-electrode gap, with an estimated fluence at the gap center of $\sim 1 \times 10^{15} \mathrm{~cm}^{-2}$, i.e., two orders of magnitude below the graphitization threshold.

An electrical characterization of the fabricated structure was performed by means of current-voltage measurements (Fig. 2a) between the two independent electrodes (experimental parameters: 2-terminal configuration, $0-500 \mathrm{~V}$ voltage range, $3 \mathrm{~s}$ source time and $2 \mathrm{~V}$ voltage step). The measured current was lower than $100 \mathrm{pA}$ at $\pm 100 \mathrm{~V}$ applied bias, thus confirming the mutual insulation of the electrodes through the annealed diamond matrix and indicating a negligible surface leakage current. Particularly, such result evidenced that the thermal annealing effectively prevented the residual radiation damage associated with stray ions implanted in the electrodes gap from affecting the overall insulation properties of the material.

On the other hand, at applied voltages larger than $+400 \mathrm{~V}$ an abrupt current increase to few hundreds of nA was observed, and the current reached values of tens of $\mu \mathrm{A}$ at $+500 \mathrm{~V}$. Such phenomenon was repeatable and non-destructive for the sample under test. EL was observed with the transition to the high-current regime along a straight path connecting two buried electrodes (Fig. 2b), suggesting that the sudden current increase is caused by the injection of charge carriers at localized sites at the interface between diamond and graphite. Furthermore, the $I-V$ characteristic showed an hysteretical behavior, with higher current values at decreasing bias than those exhibited at increasing voltages. The reported observations indicate a different behavior from what reported in a previous work on the characterization of graphitic channels in diamond, where the $I-V$ characteristic was interpreted in terms of an avalanche breakdown [5]; therefore, further
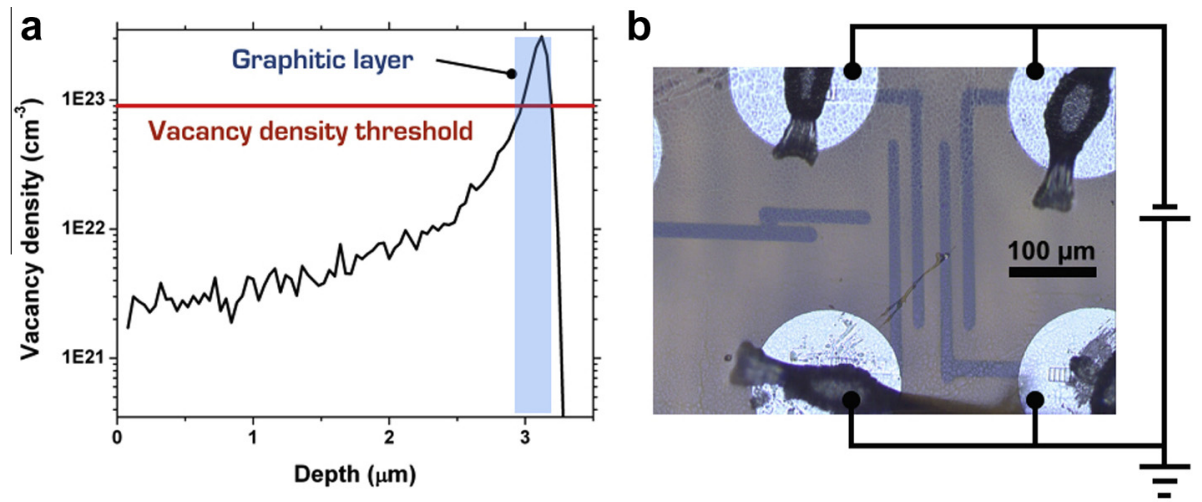

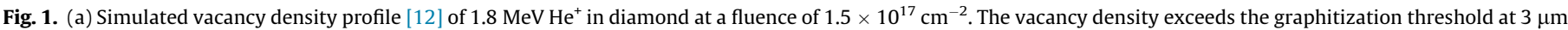

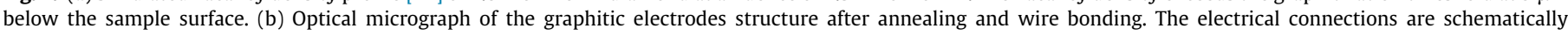
presented. 

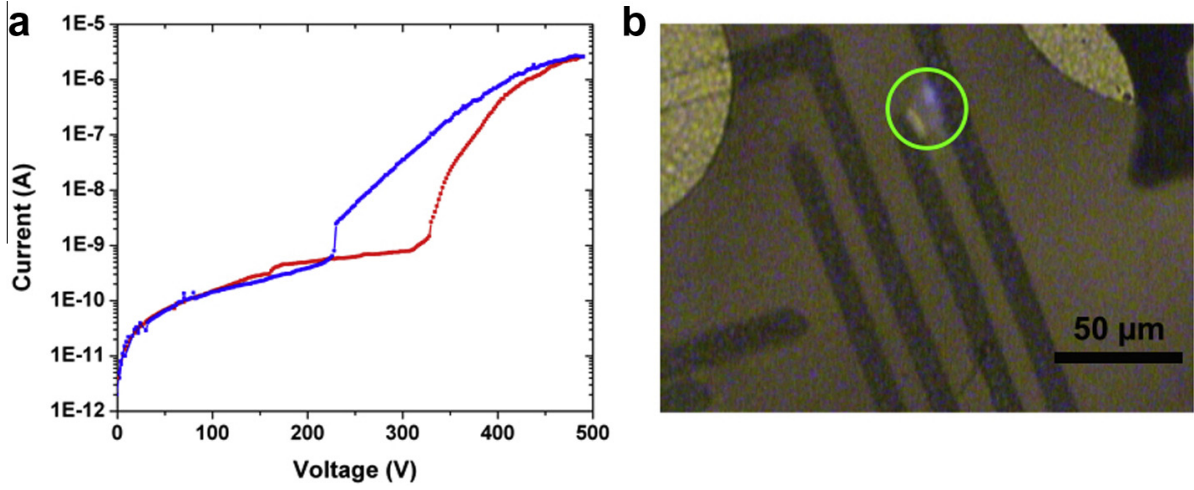

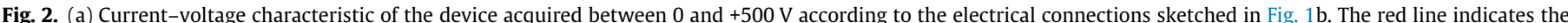

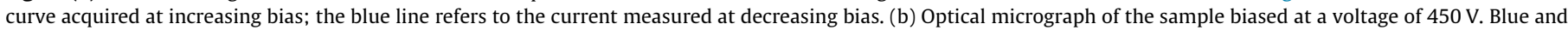

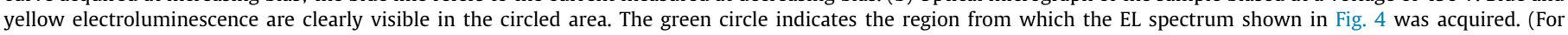
interpretation of the references to color in this figure legend, the reader is referred to the web version of this article.)

investigations will be necessary to unequivocally interpret the charge conduction mechanism in the device under test.

\subsection{Optical characterization}

The electric current flowing between the buried channels was exploited to provide the electrical excitation of the color centers in the inter-electrode gap. A bright EL emission, visible to the bare eye, typically appeared at currents above $\sim 100 \mathrm{nA}$ and increased in intensity at increasing bias. In Fig. $2 \mathrm{~b}$, an optical micrograph of the inter-electrode region of the sample taken at a bias voltage of $+450 \mathrm{~V}$ is shown, where EL is clearly visible, and the presence of different spectral components of the emitted light is apparent.

In order to clarify the emission properties of the electroluminescent device and to identify with higher spatial resolution the light-emitting regions of the structure, an EL mapping of the sample was performed by adapting a home-built confocal photoluminescence microscopy setup (Fig. 3a) [13]. The sample was mounted on a remotely-controlled three-axis piezo-electric stage, with a scan area of $100 \times 100 \mu \mathrm{m}^{2}$ and a nanometer positioning accuracy, and was raster-scanned to image the EL properties of the structure under test. The induced luminescence was collected by a $100 \times$ air objective (numerical aperture N.A. $=0.9$ ) and then focused with an achromatic doublet into a graded-index multimode optical fiber, which both provided an optical connection to the detection system and acted as the pinhole aperture for the confocal system. The detection system consisted of a photon-counter based on one Si-single-photon-avalanche photo-diode (SPAD) Perkin-Elmer SPCARQ14-FC operating in Geiger mode; a digital counter recorded the total counts detected by the SPAD, enabling the measurement of the total EL intensity for each pixel.

\section{Results}

An EL map acquired at a bias voltage of $450 \mathrm{~V}$ is shown in Fig. 3b. The encoded color scale displays for clarity a maximum emission rate value of $5 \times 10^{4}$ counts per second (cps), each count corresponding to the detection of a single photon; however, the values recorded at the center of the bright spot reached values larger than $10^{6} \mathrm{cps}$. The map clearly shows that the electroluminescent region corresponds to a localized conduction path connecting the buried electrodes (highlighted by the dashed black lines in Fig. 3b). The conductive path corresponds to the bright region highlighted in the optical micrograph in Fig. 2 and originates at the endpoint of the grounded electrode, where the sharpening of the buried graphitic channel increases the local electric field as an effect of the tip geometry.

With the purpose of performing spectroscopic measurements, the radiation from the light-emitting region highlighted by the green circle in Fig. 2, comprising most of the EL signal was collected with a Jobin Yvon Raman micro-spectrometer equipped with a CCD Andor "DU420A-OE" detector. As the light emission is not stimulated by a localized standard laser excitation, but instead is induced by the electrical current flowing between the electrodes, the collected light is not spatially limited to the focal point of the experimental setup and the spatial resolution for the spectral measurement was estimated to be of $\sim 10 \mu \mathrm{m}$. A typical EL spectrum is shown in Fig. 4. The two dips at $633 \mathrm{~nm}$ and at a

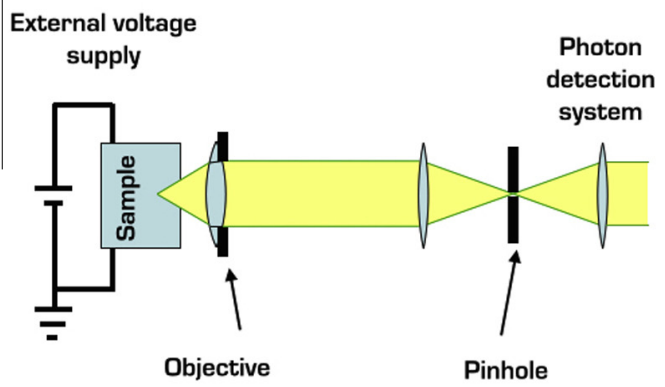

b

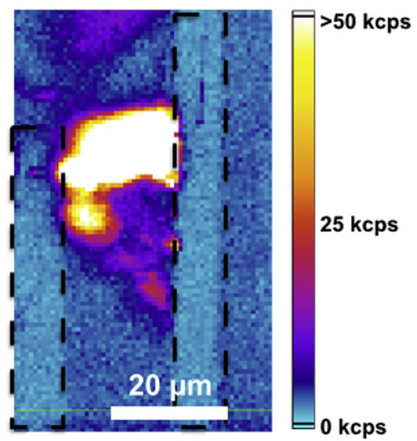

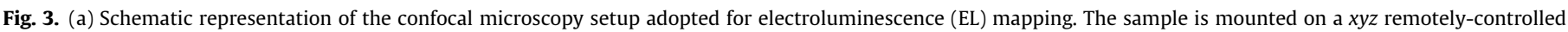

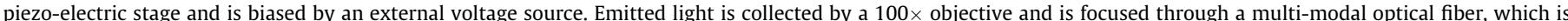

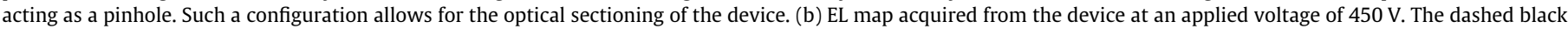
lines identify the relative position of the graphitic electrodes. 


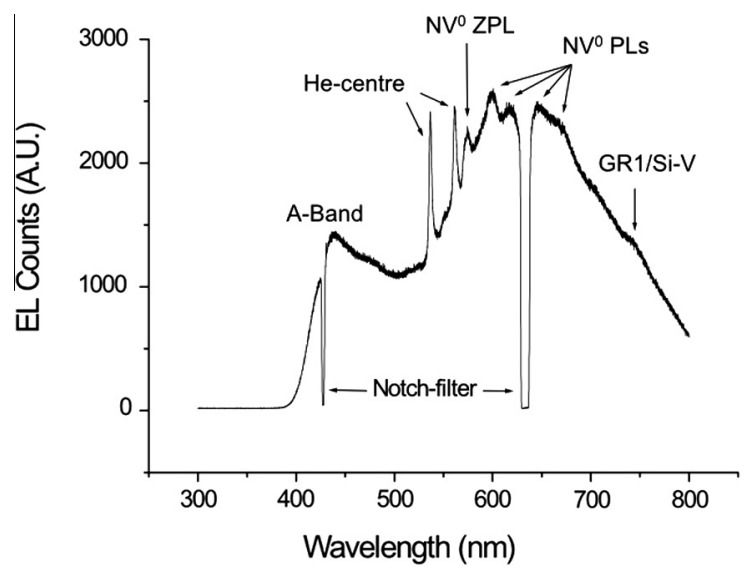

Fig. 4. EL spectrum from the yellow region indicated by the green circle in Fig. $2 \mathrm{~b}$. The following features are highlighted: A-band; He-center; $\mathrm{NV}^{0}$ and (tentatively) GR1 or Si-V. A notch filter present in the spectrometer creates two dips at 428 and $633 \mathrm{~nm}$.

$428 \mathrm{~nm}$ are instrumental artifacts caused by the presence of a notch filter mounted in the spectrometer, which attenuates the laser excitation when the setup operates in its usual configuration. Starting from lower wavelengths, different features can be identified in the EL spectrum. Firstly, a $\sim 100 \mathrm{~nm}$ broad peak centered at $435 \mathrm{~nm}$ is visible. The broad peak is usually referred as the Aband and its origin is commonly attributed to radiative carriers recombination at extended lattice dislocations. Evidence of the Aband was found in previous EL experiments in diamond P-I-N junctions $[11,14,15]$. Therefore, the observation of the $435 \mathrm{~nm}$ broad peak proves that the injected charge carriers are interacting with electronic states within the bandgap [14]. Secondly, the two sharp peaks at $536.3 \mathrm{~nm}$ and $560.5 \mathrm{~nm}$ are attributed to a helium-related defect previously observed in cathodoluminescence [5,15], consistently with the fact that $\mathrm{He}^{+}$was employed for the micro-fabrication process and stray ions could easily have been implanted in the inter-electrode gap region. Thirdly, the peak at $575 \mathrm{~nm}$ and its replica at higher wavelengths respectively correspond to the zero-phonon-line and phonon sidebands of the well-known $\mathrm{NV}^{0}$ center, as extensively observed in cathodoluminescence and EL measurements $[5,9,10]$. Finally, a small peak emerging from the background at $\sim 738-740 \mathrm{~nm}$ can be tentatively attributed to the Si-V color centers [15], since Si could be present as a contaminant in the CVD chamber, and it has been reported to be active in EL in previous works [16]. Alternatively, the peak could be ascribed to the general radiation center GR1 $(741 \mathrm{~nm})$, which is associated with radiation-induced vacancies. Although a direct observation in electroluminescence has not been reported so far to our knowledge, the emission from GR1 has been observed in cathodoluminescence regime [15] where a similar excitation process through the injection of electrodes in the diamond dielectric is taking place. Finally, it is worth noting the absence of the radiation B-band $(770 \mathrm{~nm})$ associated with radiation damage and reported in previous EL works [10]. Despite the spatial resolution of the experimental setup was not sufficient to provide a position-dependent analysis, the micrograph in Fig. $2 \mathrm{~b}$ shows that the contributions of the A-band and of the He-related center vary from region to region: areas with a greater contribution from the A-band are clearly identified by the blue light, while regions dominated by the emission at longer wavelength, where no contribution is associated with extended lattice dislocations, appear as yellow.

Such observation is consistent with the injection of charge carriers in the inter-electrode gap at a specific position, where EL occurs, at the graphite-diamond interface. Particularly, the presence of lattice dislocations might indicate a position where the carriers injection could require a lower applied voltage than the surrounding regions.

\section{Conclusions}

A structure consisting of parallel buried graphitic electrodes in single-crystal diamond was fabricated with DIBL with a $1.8 \mathrm{MeV}$ $\mathrm{He}^{+}$scanning microbeam. The current flowing in the gap region between the buried electrodes has been exploited to stimulate EL from color centers located in the above-mentioned region. EL mapping highlighted that the emission occurs along localized paths between the electrodes. The EL spectrum exhibited light emission from dislocation clusters (A-band), $\mathrm{NV}^{0}$ centers and helium-related defects associated with the ion implantation. The results presented in this work confirm that it is possible to electrically excite color centers by means of charge injection from graphitic non-rectifying electrodes, although a deeper investigation of the electrical properties of the electrodes geometry under test will be required to shed light on the charge injection and the charge transport properties of the device.

Furthermore, the results highlight the possibility of electrically exciting He-related defects, which are characterized by appealing photophysical properties, i.e. high emission rates, sharp spectral emission, negligible phononic sidebands. The possibility to identify and electrically stimulate isolated He-related defects might open to the study of new electroluminescent single-photon sources in diamond for applications in photonics and quantum information.

\section{Acknowledgements}

This work is supported by the following projects: FIRB "Futuro in Ricerca 2010" (CUP code: D11J11000450001) funded by the Italian Ministry for Teaching, University and Research (MIUR); “A.Di.N-Tech." project (CUP code: D15E13000130003) funded by the University of Torino and Compagnia di San Paolo in the framework of the 'Progetti di ricerca di Ateneo 2012' scheme; EMRP project 'EXL02-SIQUTE', jointly funded by the EMRP participating countries within EURAMET and the European Union.

\section{References}

[1] J.O. Orwa, A.D. Greentree, I. Aharonovich, A.D.C. Alves, J. Van Donkelaar, A Stacey, S. Prawer, J. Lumin. 130 (2010) 1646-1654.

[2] F. Picollo, D. Gatto Monticone, P. Olivero, B.A. Fairchild, S. Rubanov, S. Prawer, E. Vittone, New J. Phys. 14 (2012) 053011.

[3] P. Olivero, J. Forneris, M. Jakšić, Ž. Pastuović, F. Picollo, N. Skukan, E. Vittone, Nucl. Instr. Meth. B 269 (2011) 2340.

[4] R. Kalish, S. Prawer, Nucl. Instr. Meth. B 106 (1995) 492.

[5] A.A. Gippius, R.A. Khmelnitskiy, V.A. Dravin, S.D. Tkachenko, Diamond Relat. Mater. 8 (1999) 1631

[6] J. Forneris, V. Grilj, M. Jakšic, A. Lo Giudice, P. Olivero, F. Picollo, N. Skukan, C. Verona, G. Verona-Rinati, E. Vittone, Nucl. Instr. Meth. B 306 (2013) 181.

[7] F. Picollo, S. Gosso, E. Vittone, A. Pasquarelli, E. Carbone, P. Olivero, V. Carabelli, Adv. Mater. 25 (2013) 4696.

[8] S. Prawer, A.D. Devir, L.S. Balfour, R. Kalish, Appl. Opt. 34 (1995) 636.

[9] N. Mizuochi, T. Makino, H. Kato, D. Takeuchi, M. Ogura, H. Okushi, M. Nothaft, P. Neumann, A. Gali, F. Jelezko, J. Wrachtrup, S. Yamasaki, Nat. Photonics 6 (2012) 299.

[10] A. Lohrmann, S. Pezzagna, I. Dobrinets, P. Spinicelli, V. Jacques, J.-F. Roch, J Meijer, A.M. Zaitsev, Appl. Phys. Lett. 99 (2011) 251106.

[11] S. Koizumi, K. Watanabe, M. Hasegawa, H. Kanda, Science 292 (2001) 1899.

[12] J.F. Ziegler, M.D. Ziegler, J.P. Biersack, Nucl. Instr. Meth. B 268 (2010) 1818.

[13] D. Gatto Monticone, P. Traina, E. Moreva, J. Forneris, P. Olivero, I.P. Degiovanni, F. Taccetti, L. Giuntini, G. Brida, G. Amato, M. Genovese, New J. Phys. 16 (2014) 053005.

[14] C. Manfredotti, F. Wang, P. Polesello, E. Vittone, F. Fizzotti, A. Scacco, Appl Phys. Lett. 67 (1995) 3376.

[15] A.M. Zaitsev, Optical Properties of Diamond - Data Handbook, Springer, New York, 2001.

[16] A.A. Melnikov, A.V. Denisenko, A.M. Zaitsev, V.S. Varichenko, V.P. Varnin, I.G. Teremetskaja, V.A. Laptev, A. Yu Palyanov, W.R. Fahrner, B. Burchard, in: S. Saito, N. Fujimory, O. Fukunaga, M. Kamo, K. Kobashi, M. Yoshikawa (Eds.), Advances in New Diamond Science and Technology, MYU, Tokyo, 1994. 